\title{
TRACMASS - A mass conserving trajectory code for ocean and atmosphere general circulation models
}

Aitor Aldama Campino1('), Kristofer Döös ${ }^{1}$, Sara Berglund¹, Dipanjan Dey¹, Joakim Kjellsson2,3, and Bror Jonsson 4

\section{What's TRACMASS?}

- TRACMASS is a Lagrangian trajectory code for ocean and atmospheric general circulation models (GCM).

- The TRACMASS scheme is mass conserving within the grid cell in the same way as the GCM.

- The code makes it possible to estimate water paths, Lagrangian stream functions (barotropic and overturning), exchange times, etc.

- TRACMASS has been set up to run with velocities integrated with models such as NEMO, ROMS, MOM, ECMWF-IFS (ERA 5 and EC-Earth).

Example of Lagrangian stream functions
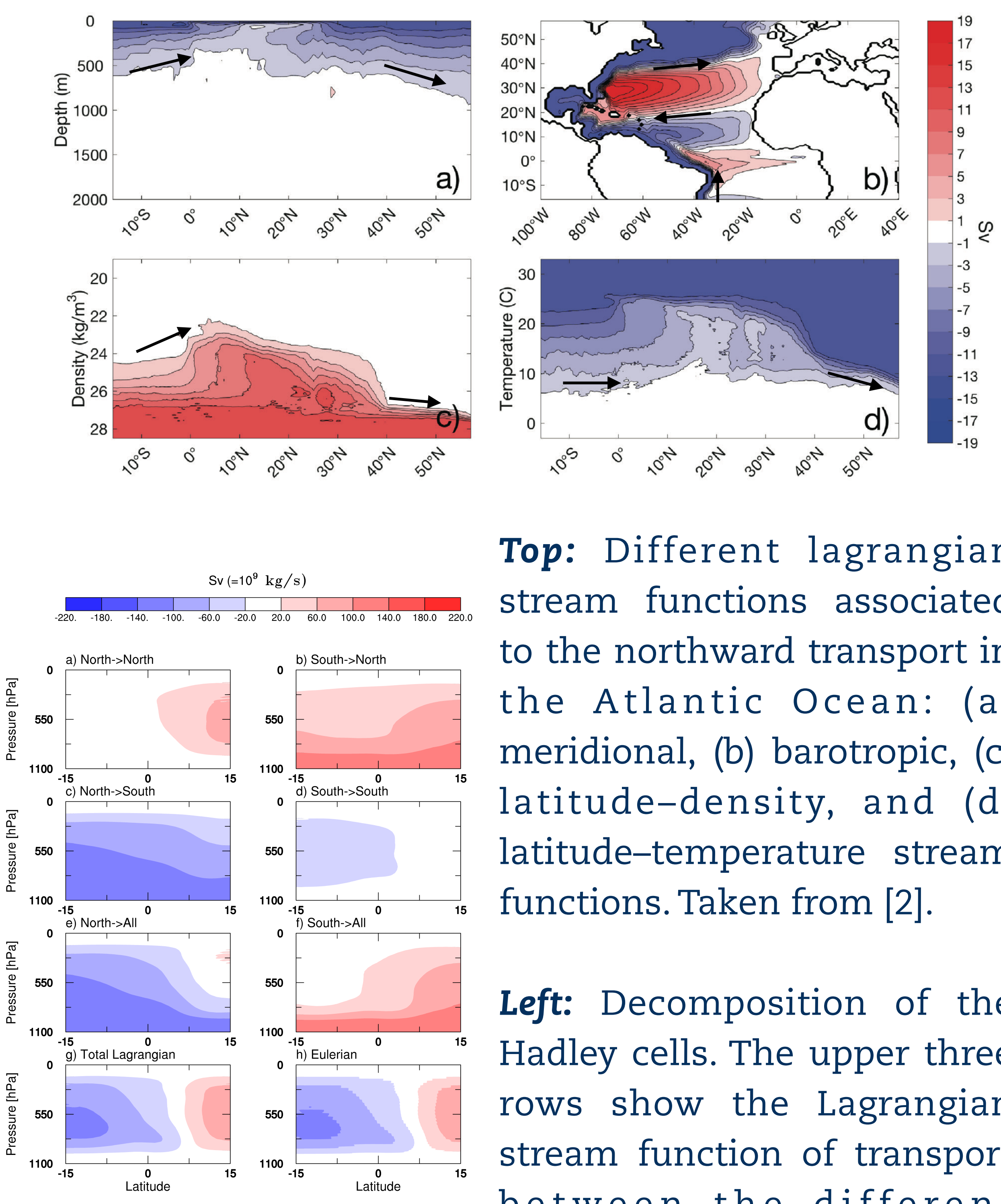

Top: Different lagrangian stream functions associated to the northward transport in the Atlantic Ocean: (a) meridional, (b) barotropic, (c) latitude-density, and (d) latitude-temperature stream functions. Taken from [2].

Left: Decomposition of the Hadley cells. The upper three rows show the Lagrangian stream function of transport between the different latitudes.

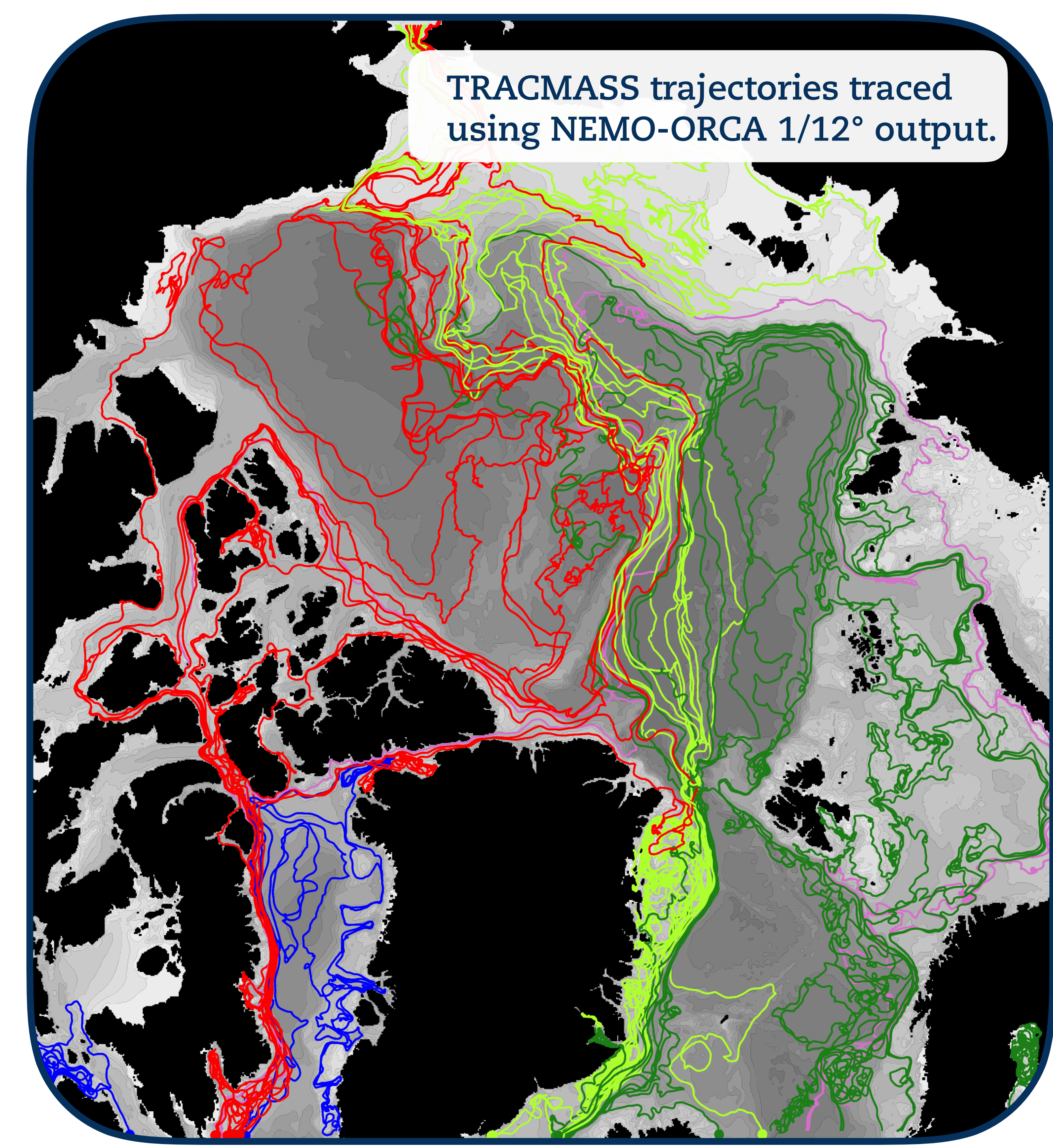

\section{Quick start}

1- Set up TRACMASS:

Enter the tracmass directory and copy the template Makefile

cd tracmass

cp Makefile_tmpl Makefile

Modify the Makefile to fit your system. You will also need to configure how TRACMASS should find the netCDF libraries, if at all. Then you can run the make command.

make

\section{2- Running a test case:}

Run the test case for TRACMASS by letting PROJECT and CASE be "theoretical" in the Makefile (which is the default).

In this case, TRACMASS will use a simple oscillating velocity field to trace trajectories. You can run this case by setting

. /runtracmass

Get the code here

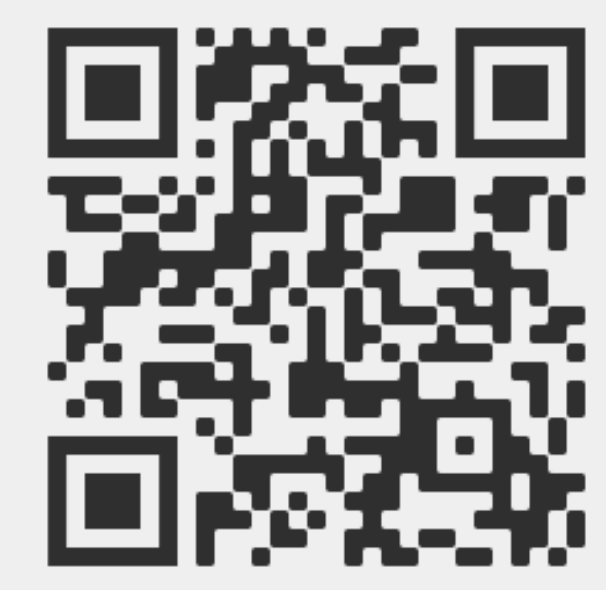

GitHub repository: https://github.com/TRACMASS/tracmass 


\section{Mass conservation in TRACMASS}

- TRACMASS uses mass fluxes $[\mathrm{kg} / \mathrm{s}]$ instead of velocities $[\mathrm{m} / \mathrm{s}]$ :

$$
U_{i, j, k}^{n}=\rho_{i, j, k}^{n} \Delta y_{i, j} \Delta z_{i, j, k} u_{i, j, k}^{n}
$$

which works with any vertical coordinate ( $\mathrm{z}, \mathrm{p}, \mathrm{z}^{*}$, hybrid, $\sigma$ levels, etc.) and both hydrostatic and non-hydrostatic settings.

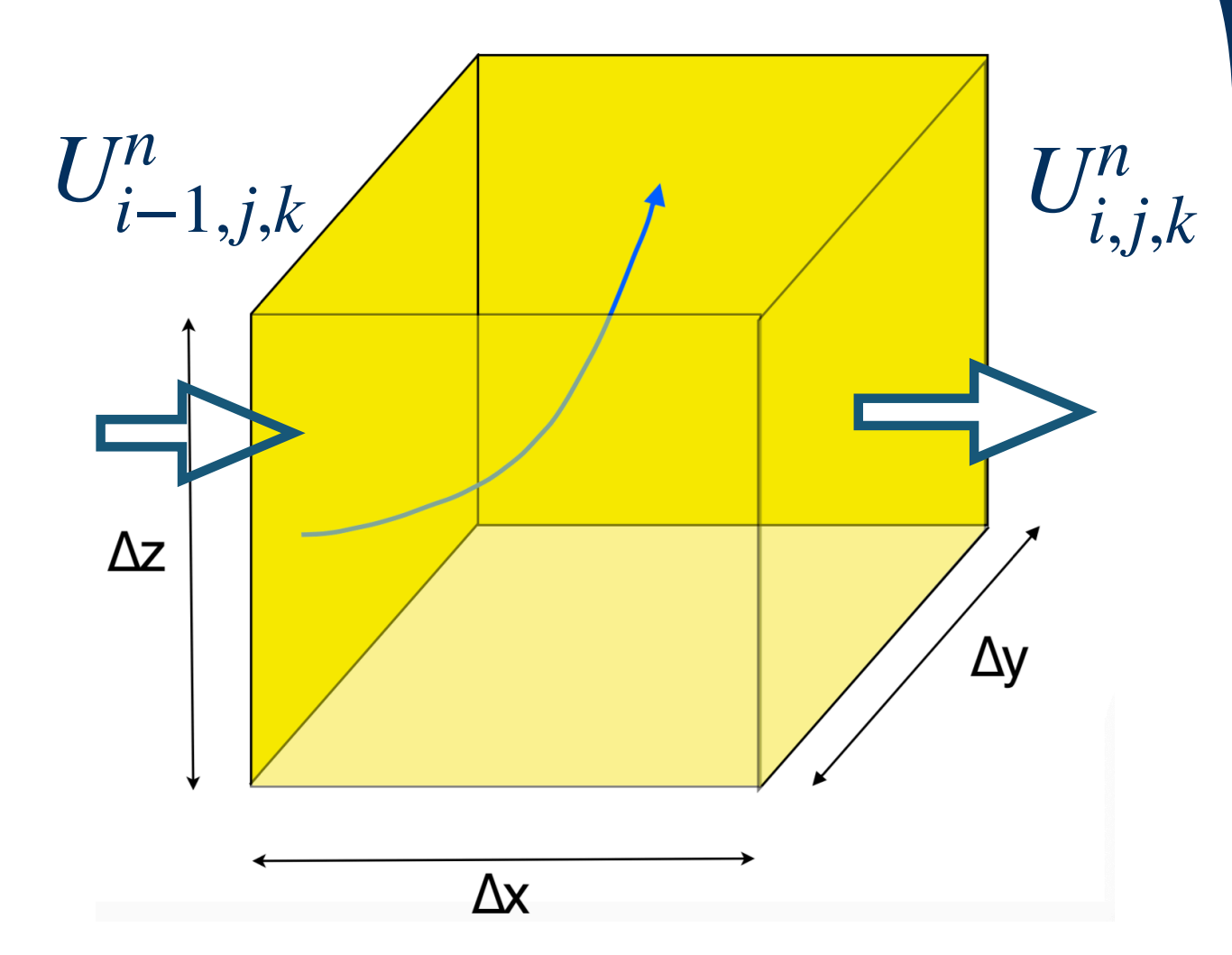

- The vertical mass fluxes are computed from the continuity equation:

$$
\frac{\partial M_{i, j, k}}{\partial t}+U_{i, j, k}-U_{i-1, j, k}+V_{i, j, k}-V_{i, j-1, k}+W_{i, j, k}-W_{i, j, k-1}=0
$$

- Bilinear interpolation (space and time) within the grid box of the mass transport.

- Analytical trajectory solution through the grid box.

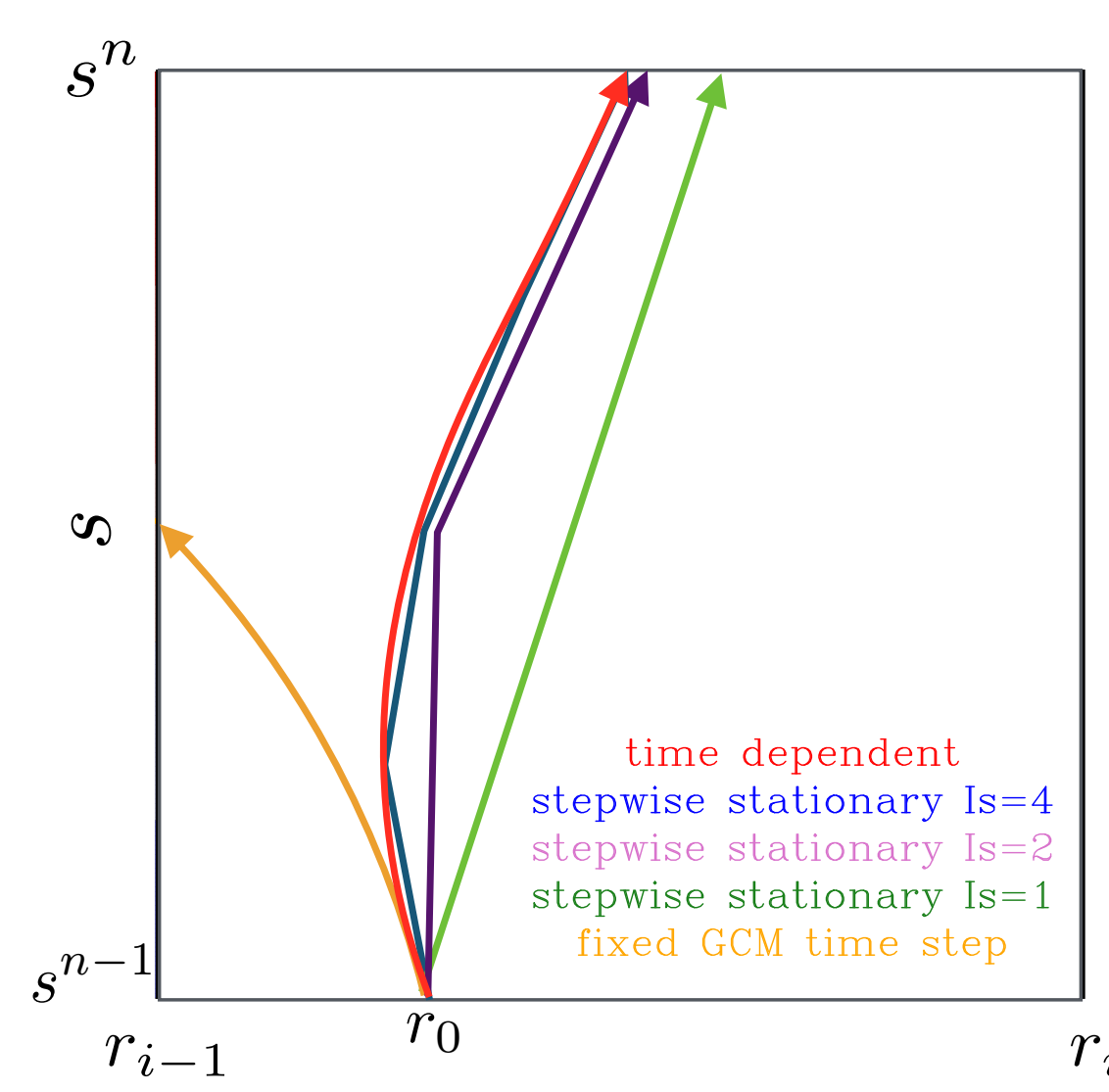

More examples: Atmosphere

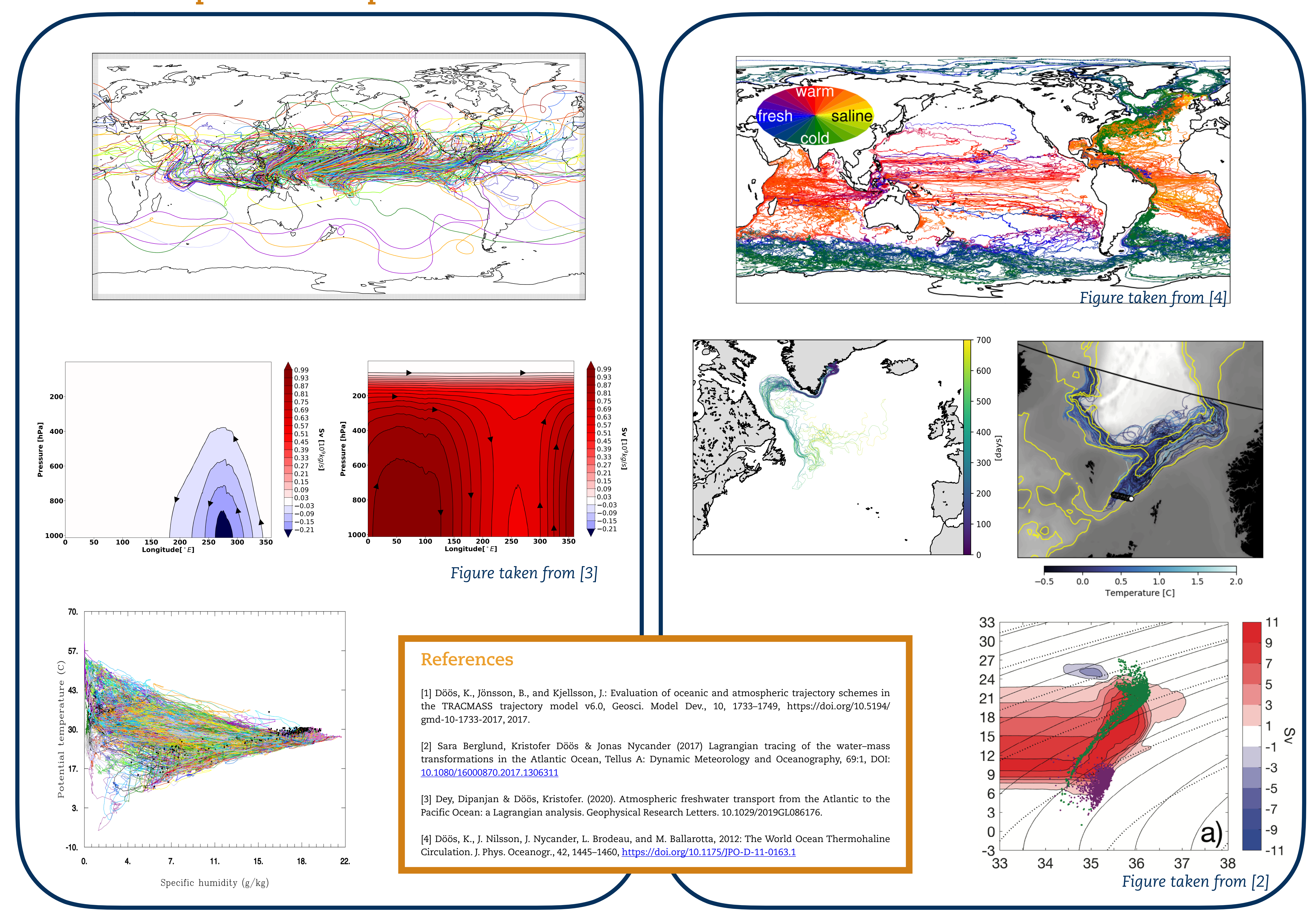

